\title{
Quality control by Infrared Thermography of the infusion manufacturing process of composite automotive specimens
}

\author{
by P. Venegas*, I. Ortiz de Mendibil**, A. Montero* and J. Aurrekoetxea** \\ * Centro de Tecnologías Aeronáuticas, Parque Tecnológico de Álava, Spain, pablo.venegas@ctaero.com \\ **Escuela Politécnica Superior de Mondragon Unibertsitatea, Arrasate, Spain, imendibil@mondragon.edu
}

\begin{abstract}
Arial, 9pt, bold)
In this study the main defects appearing in composite manufacturing by infusion process are investigated to develop specific quality control routines. The technology employed to analyze this process and carry out quality controls is infrared thermography which, due to the special configuration of infusion manufacturing, allows an on-line monitoring of the whole process.

This study was conducted at laboratory level with different composite materials, determining the thermal behaviour of the zones undergoing anomalies. This way a complete procedure for anticipating defect initialization and stopping the manufacturing process in early stages was defined, enabling repair actions and saving time and costs.
\end{abstract}

\section{Introduction}

Structural reinforced plastics are catching the interest of the industrial market in general, and automotive industry in particular, due to their high specific mechanical properties. This increased use of composite materials has also led to the appearance of new manufacturing processes. One of these techniques is the Vacuum Assisted Resin Infusion; this flexible process allows the production of large parts keeping the manufacturing costs low. However, this production technique is heavily influenced by the human factor as some key steps, such as the construction of the vacuum bag, are performed manually. An inspection technique is desirable in order to assure the quality of the process.

Another incipient technology at present is infrared thermography, which allows the visualization of the temperature on a surface accurately and without contact. Through theorems and physical principles we can convert measurements of the infrared radiation into temperature measurements. However, the field of application of this technology is an extension that goes far beyond simply making temperature measurements, covering both industrial applications and research and development.

These two premises together with the innovative idea of the future industry, this is the so call Industry 4.0 or the fourth industrial revolution, which demands a number of new automation instruments, data exchange and manufacturing technologies, represent an excellent opportunity to advance in the proposed line of research. Industry 4.0 will facilitate the vision and execution of a "Smart Factory". Within the modular structured Smart Factories of Industry 4.0, cyberphysical systems monitor physical processes, create a virtual copy of the physical world and make decentralized decisions. In contribution to this new term of industry it is proposed to develop a quality control procedure based on IRT for infusion manufacturing real-time monitoring.

\section{Infusion manufacturing process}

\subsection{General description}

There exist many different manufacturing methods for composite materials, each of which has its own characteristics being more suitable to a particular type of structure parts according to their final application. The simplest methods of manufacturing composite material, and also the most widely used, consist of a first stage of stacking dry fiber layers on an open mould in specific number and relative orientation to produce the desired final resistance model. Then the binder matrix material is poured on to the mould, impregnating dry fibers. Finally the group of fibers and matrix is subjected to a thermal cycle under overpressure conditions within autoclave, which causes the material to be consolidated by curing and acquires the final strength.

The main advantage of forming in autoclave is the flexibility to process different families of materials. Any polymeric material may be processed in autoclave as long as the cure cycle is within the limitations of temperature and pressure of the autoclave. However, it also has several limitations among which the most important is that derived from auotoclave size and associated operating costs. That is why this manufacturing method results uneconomical for manufacturing cases of large number of small pieces. As a result of this situation there have been a variety of alternative manufacturing methods that do not require autoclave, where curing of the materials is performed outside an autoclave. These include the infusion process. 


\subsection{Defects in infusion manufacturing}

As with all manufacturing methods, the infusion process presents certain number of inherent potential defects that can arise if the necessary measures are not taken during processing. Note that currently this manufacturing method is a largely manual process, where both the stacking of fabrics and the placing of the vacuum bag is made by hand, ensuring proper sealing of the system by visual inspection.

The main defects that can be originated in this manufacturing process are:

1. Excessive porosity: It occurs when the vacuum conditions are lost within the system generally by air intake from the periphery, which is sealed with adhesive caulk. The air intake can also occur by point punctures the bag silicone contact accidentally with other sharp objects. The air inlet in the system during curing of the resin, results in a process of incorrect curing causing the final properties of the material does not meet specifications.

2. Dry areas: It occurs when the resin is not capable of properly mixing with the reinforcing material due to a bad resin flow, which causes the path followed by the fluid does not reach all areas of the mould. Generally this defect occurs in areas with complex geometries with changes in orientation and small radius of curvature that obstruct the penetration of the resin.

3. Odd material inclusions: It occurs when during stacking of dry fabrics some other foreign element not included in the production process is introduced accidentally in the mould. Generally these elements tend to be small pieces of consumables such as protective films, cutter, etc. The presence of foreign material within the composite material makes the resistive properties of the final material not correspond to the initial specifications appearing vulnerabilities in these areas.

\section{IRT Monitoring procedure}

In this study a series of tests at laboratory were conducted to determine the ability of IRT to provide useful information to manage infusion manufacturing process. The specific equipment and test configuration which were employed in these campaigns is described below.

\subsection{Equipment \& Test set-up}

Within the test environment we found two clearly different equipment. On the one hand we have the system used to carry out the manufacturing process by infusion of composite parts, and on the other hand we have the system used to monitor the status and evolution of the process by IRT.

Basically the system for manufacturing composite parts consists of a mould heated by a system of oil that is circulated through tubes. On the heated mould dry fabrics are stacked sequentially in a specific order and orientation and then are covered with a protective film and a mesh grid that facilitates the flow of resin. Two thermocouples are set in the top of these materials to keep track of the temperature during the process and finally everything is covered with a nylon bag that seals the system and allows vacuum conditions to be created inside. For the vacuum conditions to be set a hole is practiced in the bag for air to be removed by a pump. This hole is also used to empty the excess of resin at the end of the liquid flow path. (See figure 1).
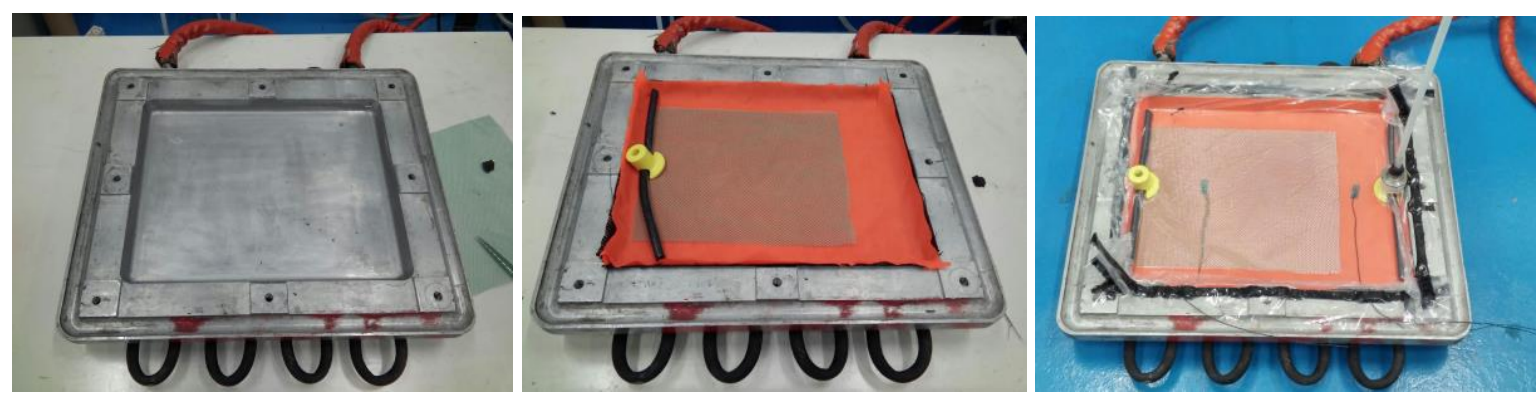

Fig. 1. Empty heated mould (left), mould and materials placed (centre) and manufacturing system at vacuum conditions (right).

Thermographic monitoring system basically consists of an IRT camera mounted on a tripod and oriented towards the free surface of the infusion mold approximately $75^{\circ}$ inclination and located at a distance of 1 (see figure 2 ). Infrared images are acquired using a FLIR SC5500 camera. This camera is equipped with a cooled Indium antimonide detector that operates in the 2.5-5.1 $\mu \mathrm{m}$ waveband. The FLIR SC5500 produces thermal images of $320 \times 256$ pixels with 12 bits per pixel and a thermal sensitivity of $20 \mathrm{mK}$.

No stimulation method was used in the IRT inspections since the infusion process itself is over-heated and the material under manufacturing experiences specific changes of temperature produced in each of the manufacturing stages. IRT camera monitors the entire manufacturing process, acquiring data from the heating of the mold until the end 
of the manufacturing process in the cooling of the finished material. The temperature evolution is controlled in real time with a laptop situated near the systems.
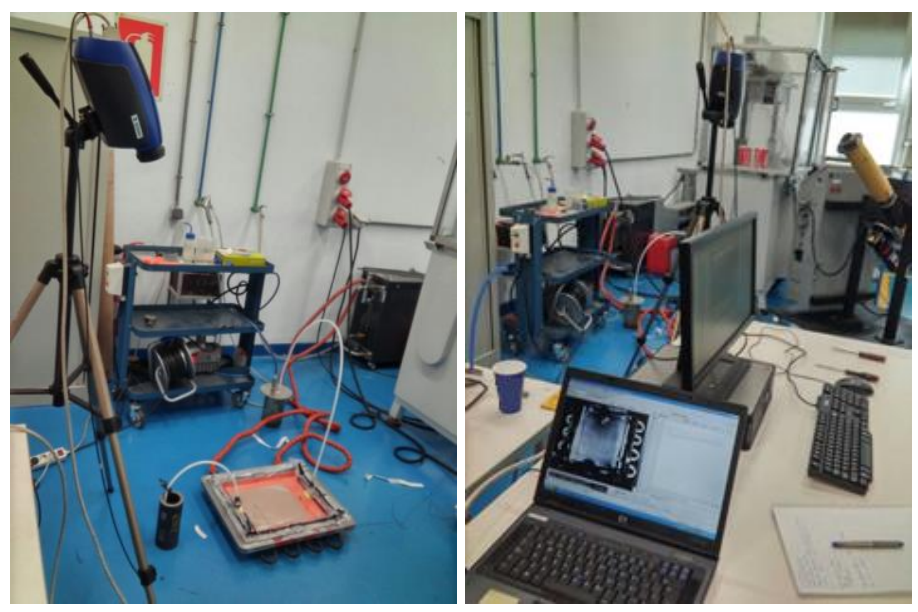

Fig. 2. IRT monitoring set-up during manufacturing process.

\subsection{IRT monitoring conditions}

As already mentioned in the previous section, the tests conducted in the laboratory have two separate systems operating in parallel: the infusion system, which is imposing the thermal conditions at every moment, and the thermographic inspection system, which must adapt to the conditions that the manufacturing system generates without interfering with it. Thus the IRT monitoring conditions are those generated in the different stages of the manufacturing infusion process, which are:

1. Preheating of mould

2. Injection of resin

3. Curing of resin

4. Cooling of finished material.

Note that all these steps are performed under vacuum conditions in the material; state which is established previously to stage 1 and kept until the end of the manufacturing process. Furthermore, in stage 3 , the action of resin flow closign is easily detected by the thermographic sensor (see figure 4) but it doesn't affect the thermal conditions of the manufactured material.

A widespread and usual method for control of conditions of the material during manufacturing by infusion process is the use of thermocouples located in the top layer of the material. This allows continuous measurement of the temperature of the material during the process in those points where thermocouples are placed. Nevertheless the number of thermocouples used must be limited to avoid affect the flow of the resin in the material due to their presence. In the test conducted two thermocouples located in the midline of the mould were used, one located closer to the entrance area of resin and the other being nearest to the exit zone of resin (see Figure 1).

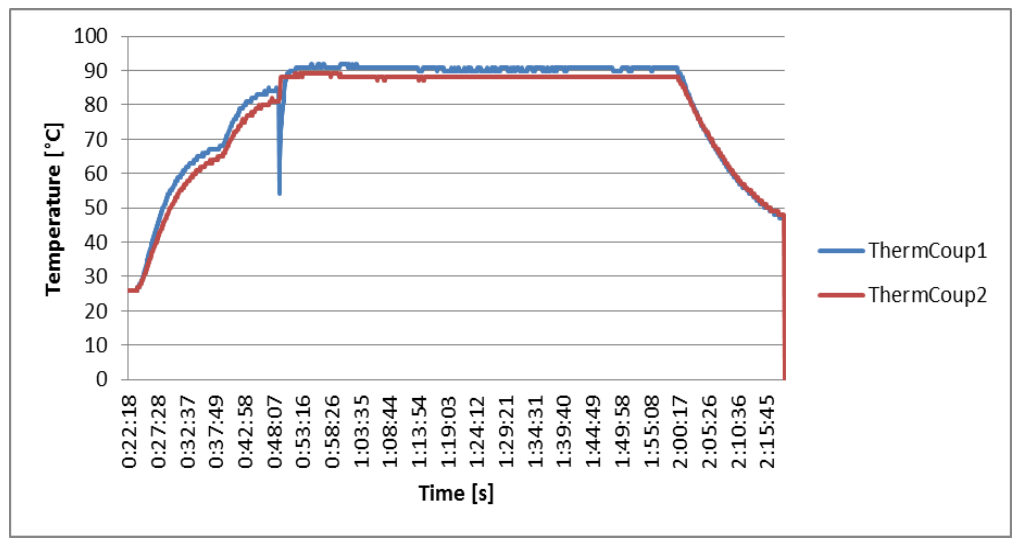

Fig. 3. Temperature evolution in two points during the infusion process measured by thermocouples. 
Main difference between thermocouple and IRT monitoring systems is based on the capacity of measuring large areas and without contact provided by IRT over the punctual and with contact measuring of thermocouples. Thanks to these aptitudes IRT can provide not only temperature evolution during the manufacturing process but also detailed thermal relations among different areas within the inspection area of interest, which may reveal presence of anomalies.

In the conducted tests IRT camera was acquiring data during all the manufacturing process. Although the camera has a maximum frame rate of $383 \mathrm{~Hz}$, full frame rate, the experiments were recorded at $10 \mathrm{~Hz}$ in order to reduce the number of acquired images. Notice that the whole infusion manufacturing process may take up to 2 hours what would produce a huge archive.

After completion of manufacturing process thermal data acquisition stops and the results are subsequently analyzed. The procedure followed consists in analyzing the temperature evolution in time, independently for each of the stages constituting the infusion process, trying to identify possible anomalous behaviors indicative of potential occurrence of defects. In this analysis task IRT data processing techniques were employed to extract as much information as possible with the aim of highlighting those signs that identify the start of some kind of defect. The types of analysis and data processing algorithms that were used are the following ones:

- Temperature time history

- Temperature gradients:

- Order: 1 and 2

- coordinates: $x, y$ and $t$

- $\quad$ Spectral analysis (Fourier)

- Modulus

- Phase

- Principal component analysis

- Statistical moments:

- Order: 3 and 4

The evaluation of the capacity of each analysis algorithm to identify the presence of a defect is made by employing the SNR metric, which relates the values of the area of interest with value of a reference area by the following equation:

$$
S N R=20 \cdot \log _{10}\left(a b s\left(\text { mean }_{\text {def }}-\text { mean }_{\text {ref }}\right) / s t d_{\text {ref }}\right)
$$

In the cases under study the reference area was considered to be the whole area of inspection.

\section{Results and discussion}

Thermal monitoring by IRT of the infusion process was developed to check the proper performance in three different stages: 1 . Analysis of the mould preheating; 2 . Analysis of the resin injection process; and 3. Analysis of the resin curing process. In order to develop a monitoring technique for a wide range of applicability, carbon as well as glass fibre composites were employed. The common mistakes appearing in infusion process were intentionally caused, such as loss of vacuum conditions, heterogeneous diffusion of the resin through fibre layers and inclusion of odd materials.

Special attention was paid to the initial phases of the manufacturing process, namely preheating and injection, because they are the phases in which greater thermal variations take place and therefore more information can be obtained from the thermographic monitoring, and also because that is in the early stages of the manufacturing process where there exists the possibility of solving present errors without causing a high increment in cost and manufacturing time.

The raw thermal data acquired directly with the thermographic system clearly identifies the phases that comprise the infusion process (see figure 4) and allows to define a preliminary strategy to carry out a thermal control of the manufacturing.
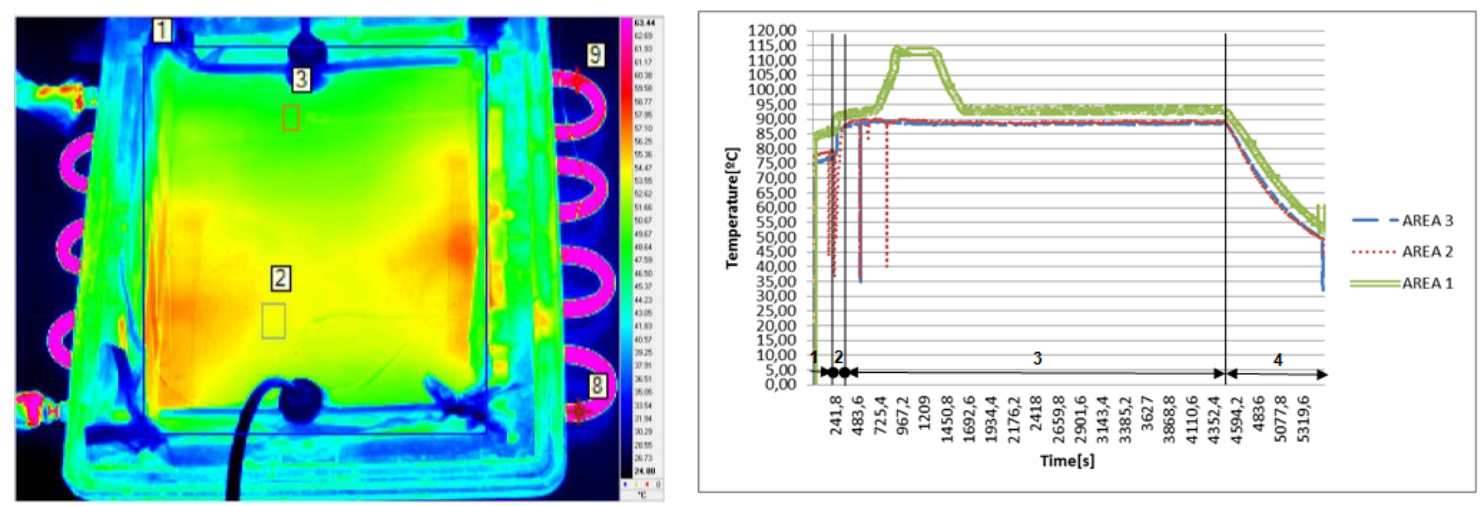

Fig. 4. Temperature evolution detected by IRT system in 3 areas of the material and mould. 
The results obtained from the analysis of each of the thermal sages of the infusion process are described in the following sections.

\subsection{Preheating stage}

The preheating stage is characterized by a generalized exponentially increase of temperature until a maximum value is reached and after which the system stabilizes. This maximum temperature is set by the control system of the manufacturing process and is important since the temperature reached in the system must be high enough to ensure activation of the curing process of the resin.

In this preheating stage the system is already under vacuum conditions, so that the most basic defect that can be originated is the loss of pressure and therefore the air inlet to the bag. Detecting this type of defect in this stage is really convenient to rectify before introducing the resin into the mould, which would effectively materialize porosity defect. This defect is usually originated from outlying areas and caused by bad sealing of adhesive paste. However, an analysis of the outlying areas to identify zones of air inlet at the periphery was not effective to carry out because those are areas where different materials with very different thermal properties are combined producing mixed results what can lead to error. It has been proved that the most effective approach is to analyze the inside area of the mould where approximately homogeneous thermal properties exist.

Zones of vacuum loss were effectively identified by analyzing the data acquired with the thermographic system (see figure 5). Those zones later became entry of air bubbles with the injection of resin. Algorithms that higher levels of detection provided in the case of loss of pressure in bag during the preheating stage were first order temperature gradients in the coordinate $t$, although other processing techniques also obtained effective detections in this defect case (see Table 1).
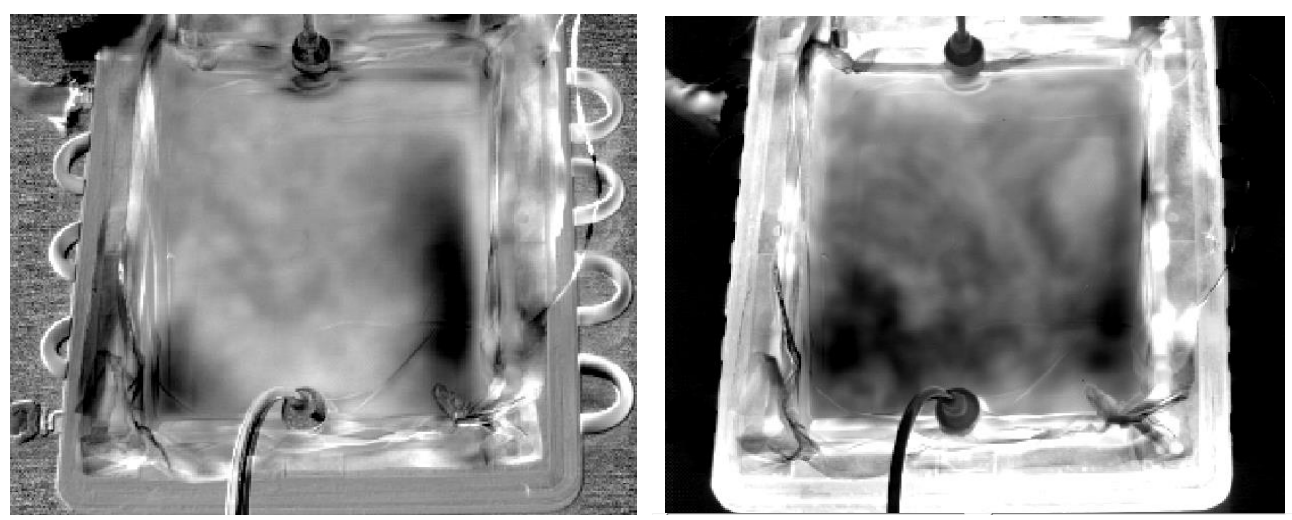

Fig. 5. Examples of identification of loss of vacuum conditions in bag during preheating stage.

The initial instants of this stage also allow to detect the presence of inclusions of foreign materials (see figure 6). However, this detection capability fades when reaching thermal equilibrium of the entire infusion system. It is not only possible to detect the presence of defects but also it is possible to characterize the type of defect by its thermal characteristics. Algorithms which have provided higher level of detection for these defects were second order gradients and the principal component analysis. It should be mentioned that in this case all processing techniques employed have revealed the presence of odd material (see Table 1).
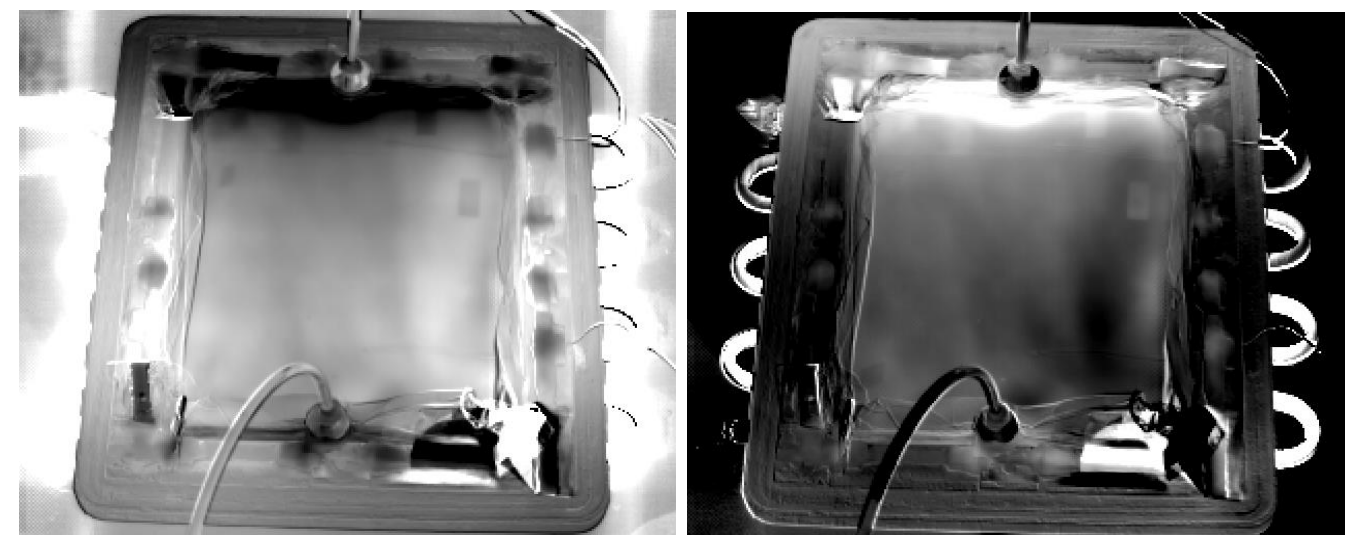

Fig. 6. Examples of odd material detection by IRT during preheating stage. 


\subsection{Resin injection stage}

This stage is the shortest one of the entire manufacturing process; it only takes a few seconds, but also it is one of the most informative stages about the quality of the manufacturing process. Areas of loss of vacuum conditions are not detected in this stage precisely because of the short duration of this phase which does not allow enough time for air to intake in the bag. On the other hand, the greatest advantage that can be obtained from the thermal process monitoring during resin injection is the direct visualization of the path followed by the resin, from entering the mould until the excess leaves the system by the sink, detecting areas which are not covered by the resin due to flow obstruction caused by sudden changes in flow orientation.

Despite the fact that this information regarding the path of the resin flow is usually not enough to avoid having to repair the part under manufacturing, or even having to discard it, the results and conclusions achieved can be used to refine the critical paths of the resin by reducing areas of curvature, designing another mould or even adding elements that improve the flow of resin in some preferred directions.

Different processing algorithms were capable of identifying the resin flow paths during injection providing the highest detection level Modulus of spectral analysis (see figure 7). Gradients of first order along time dimension also provided good efficient identification of zones with low resin flow (see table 1).

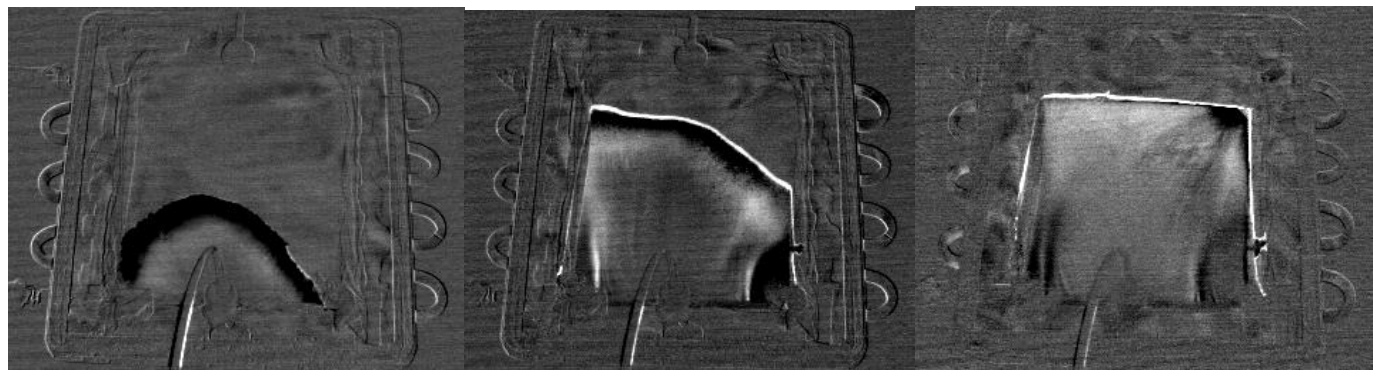

Fig. 7. Detection of resin flow path by time gradient analysis from IR data.

It is also possible in this stage to detect the presence of foreign material into the mould. Although detection is quite easy to be effectively obtained, the process of identifying the type of defect is more complicated owing to the limited time available at this stage and the limited temperature change produced by resin flow in the monitored material. In this case many processing techniques managed to effectively detect the presence of foreign materials, being the principal component decomposition the one which higher SNR provided (see Figure 8).
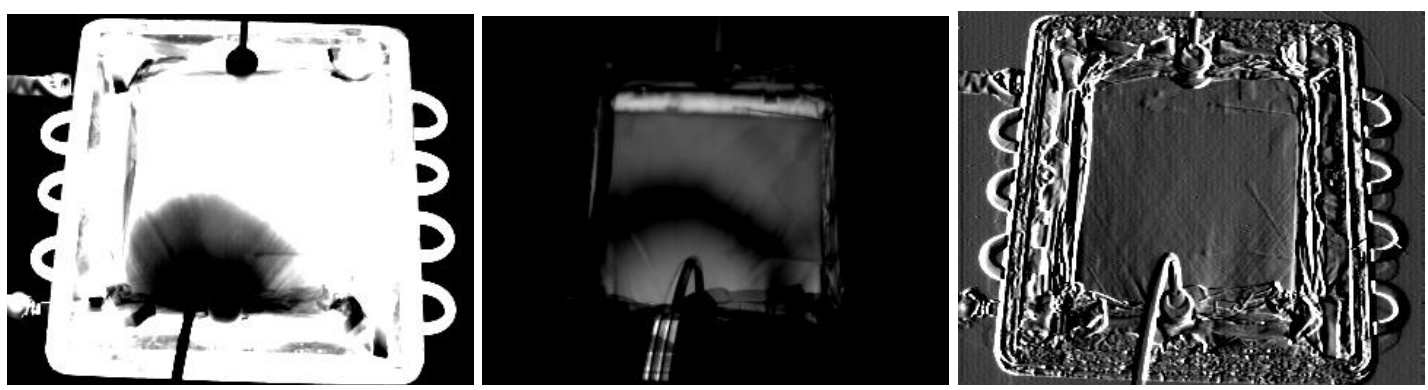

Fig. 8. Detection of foreign material inclusions during resin injection stage. Temporary analysis of temperature (left), modulus of Fourier analysis (center) and first order gradient in $x$ coordinate (right).

\subsection{Resin curing stage}

This stage is the longest one of all the infusion manufacturing process. Depending on the type of resin used in the manufacturing this stage can last more than an hour. This stage is characterized by the fact that the temperature of the system remains almost constant during the entire duration. During this process the resin hardens and material acquires its final resistive properties.

Since temperature changes are not important during resin curing stage, thermographic monitoring loses great part of its ability to provide information on the state of the material. Moreover, as the resin gets cured it also acquires hardness which reduces drastically possible air intakes preventing the movement of bubbles within the material, while the ability to solve possible detected faults is considerably limited. Therefore, the most valuable information from a quality control viewpoint is obtained from the initial instants of the curing stage and the main defect detected at this stage is the air inlet. As the thermal properties of air are so different from those of other materials involved in the manufacturing process, detection of bubbles by infrared thermography is highly effective. 
Air bubbles were very effectively detected with all the processing techniques analyzed in this study. The highest SNR was provided by the Time history analysis (see figure 9), this is, raw data directly acquired from the thermal scene.
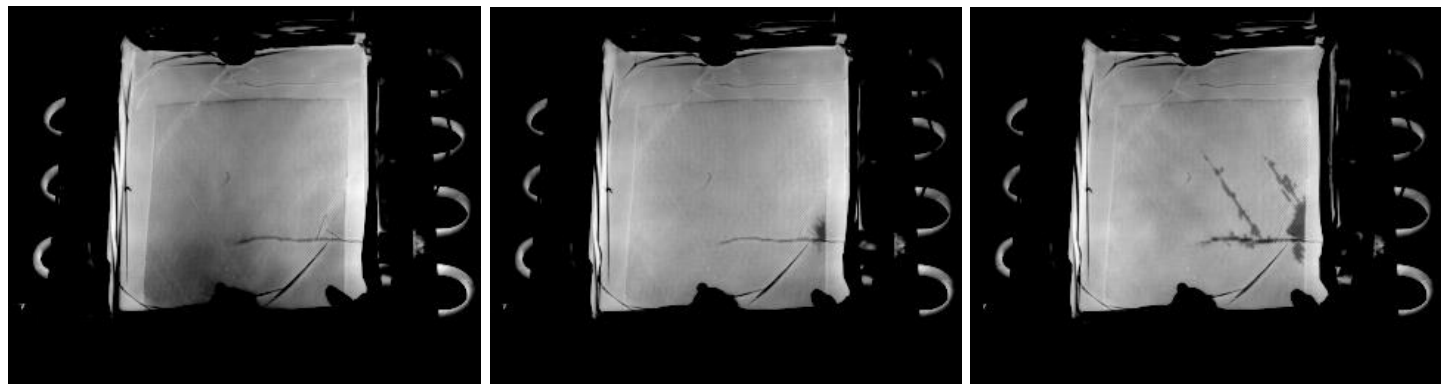

Fig. 9. Detection of air inlet and bubbles development by IRT monitoring during resin curing stage.

At this stage, although it is no longer feasible to stop the manufacturing process to remove any inserts of foreign material since the curing process is quickly activated with the resin getting hardened in a few minutes, it is still possible in the initial instants to correct possible losses of vacuum, eliminating bubbles inside the system by guiding them towards the exit zone of the remaining resin and injecting new resin from the entrance zone. After this reinjection stage it is possible to evaluate again the presence of bubbles inside the bag by IRT monitoring and check whether the defect was removed or is still present (see figure 10).
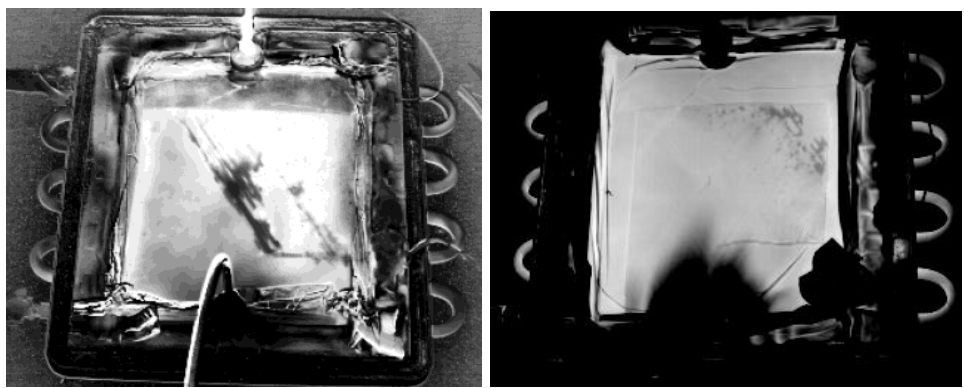

Fig. 10. Detection of air inlet by IRT (left) and monitoring of resin reinjection to remove present bubbles (right).

It was verified that the possible inserts of foreign material present in the composite were not detectable during the initial instants of the resin curing stage with the IRT monitoring system. Similarly, the final phase of the curing together with the cooling stage did not provide additional information for detecting existing defects, because the temperature remains very stable in the case of curing while the cooling process is performed naturally at a low rate without reaching the minimum temperature gradient necessary to reveal the presence of defects.

Table 1. Optimal detections produced by the different algorithms analyzed in the IRT monitoring.

\begin{tabular}{|c|c|c|c|c|c|c|c|c|c|}
\hline \multirow[b]{2}{*}{ SNR VALUES } & \multicolumn{3}{|c|}{ PREHEATING } & \multicolumn{3}{|c|}{ RESIN INJECTION } & \multicolumn{3}{|c|}{ RESIN CURING } \\
\hline & $\begin{array}{l}\text { VACUUM } \\
\text { LOSS }\end{array}$ & $\begin{array}{l}\text { DEFECTIVE } \\
\text { FLOW }\end{array}$ & $\begin{array}{l}\text { ODD } \\
\text { MATERIAL }\end{array}$ & $\begin{array}{l}\text { VACUUM } \\
\text { LOSS }\end{array}$ & $\begin{array}{l}\text { DEFECTIVE } \\
\text { FLOW }\end{array}$ & $\begin{array}{l}\text { ODD } \\
\text { MATERIAL }\end{array}$ & $\begin{array}{l}\text { VACUUM } \\
\text { LOSS }\end{array}$ & $\begin{array}{l}\text { DEFECTIVE } \\
\text { FLOW }\end{array}$ & $\begin{array}{l}\text { ODD } \\
\text { MATERIAL }\end{array}$ \\
\hline $\begin{array}{l}\text { TEMPERATURE } \\
\text { TIME HISTORY }\end{array}$ & 16,6634 & - & 12,7261 & - & 13,6866 & 14,156 & 25,9069 & $-0,9469$ & - \\
\hline \multicolumn{10}{|l|}{$\begin{array}{l}\text { TEMPERATURE } \\
\text { GRADIENTS }\end{array}$} \\
\hline ORDER 1 & 25,946 & - & 27,5754 & - & 25,2238 & 23,2384 & 22,7969 & 26,7666 & - \\
\hline ORDER 2 & 25,8332 & - & 30,4202 & - & 24,3149 & 23,1747 & 23,0409 & 25,4888 & - \\
\hline \multicolumn{10}{|l|}{ FOURIER } \\
\hline PHASE & 25,6107 & - & 22,3428 & - & 20,3016 & 17,1683 & 22,3039 & 23,444 & - \\
\hline MODULUS & 23,3029 & - & 20,6242 & - & 28,3553 & 18,4271 & 21,9723 & 24,4143 & - \\
\hline PCA & 24,4563 & - & 29,4647 & - & 15,908 & 24,6459 & 21,0005 & 23,2231 & - \\
\hline \multicolumn{10}{|l|}{$\begin{array}{l}\text { STATISTICAL } \\
\text { MOMENTS }\end{array}$} \\
\hline SKEWNESS & 2,4062 & - & 7,9046 & - & 0,0086 & 2,5195 & $-2,9223$ & 11,1048 & - \\
\hline KURTOSIS & 4,9531 & - & 5,5794 & - & $-6,0331$ & 3,0978 & $-4,0458$ & 13,1864 & - \\
\hline
\end{tabular}




\subsection{1/qirt.2016.145}

\section{Conclusions}

This study analyzed the infusion manufacturing process of composite materials and its main defects that potentially can be originated, with the aim of developing an effective control methodology to detect in advance any anomaly in the manufacturing process so that corrective action can be taken as soon as possible to avoid additional costs resulting from repair works. To do this, the tool used for monitoring activities is a basic system of infrared thermography consisted only of an infrared sensor, without additional systems of thermal stimulation, which allows thermal control of the entire surface of the material being manufactured at distance and without contact.

The main objective achieved in this study has been the validation of infrared technology as an effective tool for the infusion process control. This was obtained by conducting of controlled test campaigns at laboratory and subsequent data processing and analysis of results. The two types of composite material most widely used in the automotive industry were employed, such as polymeric materials reinforced with carbon and glass fibres, and the most common defects appearing in the method of infusion manufacturing were introduced to them, this is, losses of vacuum, defective resin flow and accidental inclusion of foreign materials. Following the acquisition of thermal data with the IRT system several processing techniques were applied and the results obtained were finally analyzed.

The main conclusions of this study relate to the evaluation criteria of the different indications that can be originated during the infusion process to be certainly considered a defect. The scope of a long series of processing algorithms applied to defects under study was defined together with the range of values that characterize them. Although there are different processing algorithms that produce optimal detections for different defects, it has been demonstrated that the temporary thermal evolution with gradient analysis are sufficient data to identify most of them effectively. This is very important because the application of a reduced number of analysis algorithms makes this analysis procedure feasible for implementation in an on-line and in real time monitoring system so that control process can be managed jointly with the rest of auxiliary manufacturing systems in an industrial global network.

Thus, as a direct result of the study, it has been developed a procedure for monitoring by IRT the infusion manufacturing process, which identifies the apparition of defects and reacts accordingly to avoid extra costs of repair. However, this procedure is based on data acquired from a finished manufacturing process. Therefore next step will be to implement this procedure in a real time control system, so as to allow interaction with the manufacturing process in taking corrective measures in case of detection of anomalies.

\section{ACKNOWLEDGEMENTS}

Authors wish to acknowledge the support of the Basque Country Regional Ministry of Industry, Innovation, Trade and Tourism for conducting this study (Project No: IE14-383 - AIRHEM III).

\section{REFERENCES}

[1] Shepard S. M., Lhota J. R., Rubadeux B. A., Wang D., and Ahmed T., "Reconstruction and enhancement of active thermographic image sequences," Optical Engineering, vol. 42, no. 5, pp. 1337-1342, 2003.

[2] Meola C.and Carlomagno G. M., "Recent advances in the use of infrared thermography," Measurement science and technology, vol. 15, no. 9, p. R27, 2004.

[3] Ibarra-Castanedo C., Gonzalez D., Klein M., Pilla M., Vallerand S., and Maldague X., "Infrared image processing and data analysis," Infrared physics \& technology, vol. 46, no. 1, pp. 75-83, 2004.

[4] Alms J, Zhou FP, Corlay C, and Suresh G. Advani "Thermal detection of air leakage in vacuum infusion processes," sampe journal 43 (1): 56-59 jan-feb 2007.

[5] Lacointe D., Vallière M., Nakousi S., Sobotka V., Boyard N., Schmidt F., Delaunay D., "Experimental determination and modelling of thermal conductivity tensor of carbon/epoxy composites". Key Engineering materials Vols. 504-506 (2012) pp1091-1096. 